\title{
Miranda
}

Revue pluridisciplinaire du monde anglophone /

Multidisciplinary peer-reviewed journal on the English-

speaking world

19 | 2019

Rethinking Laughter in Contemporary Anglophone Theatre

\section{Rachel Bouvet et Rita Olivieri-Godet, Géopoétique des confins}

\section{Françoise Besson}

\section{(2) OpenEdition}

\section{Journals}

Édition électronique

URL : http://journals.openedition.org/miranda/22308

DOI : $10.4000 /$ miranda.22308

ISSN : 2108-6559

\section{Éditeur}

Université Toulouse - Jean Jaurès

Édition imprimée

Date de publication : 7 octobre 2019

\section{Référence électronique}

Françoise Besson, «Rachel Bouvet et Rita Olivieri-Godet, Géopoétique des confins », Miranda [En ligne] 19 | 2019, mis en ligne le 15 octobre 2019, consulté le 16 février 2021. URL : http:// journals.openedition.org/miranda/22308; DOI : https://doi.org/10.4000/miranda.22308

Ce document a été généré automatiquement le 16 février 2021.

\section{(c) (i) (3)}

Miranda is licensed under a Creative Commons Attribution-NonCommercial-NoDerivatives 4.0 International License. 


\title{
Rachel Bouvet et Rita Olivieri-Godet, Géopoétique des confins
}

\author{
Françoise Besson
}

\section{RÉFÉRENCE}

Rachel Bouvet et Rita Olivieri-Godet, Géopoétique des confins (Rennes : Presses

Universitaires de Rennes, coll. "Interférences", 2018), 218p, ISBN 978-2-7535-6529-6

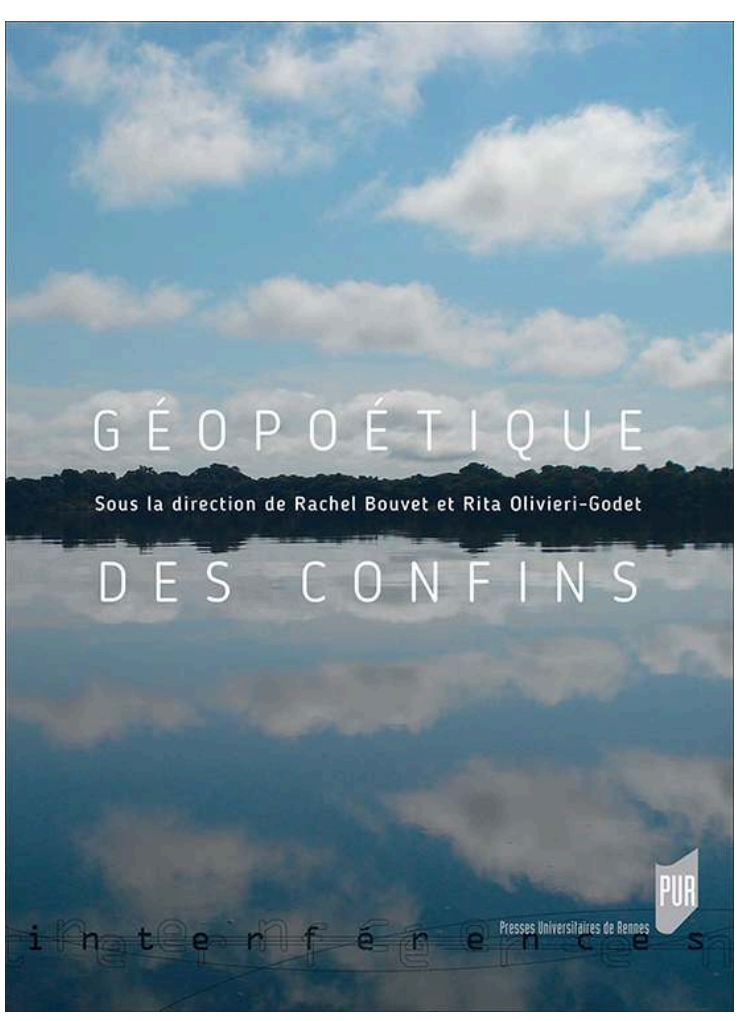


1 Cet ouvrage collectif dirigé par Rachel Bouvet et Rita Olivieri-Godet se compose de six chapitres, le premier étant un portail théorique étayé par deux autres essais, l'un sur un poème de Kenneth White et l'autre sur "'l'espace évanoui' du continent américain et l'imaginaire des confins", à partir de l'œuvre de l'écrivain-voyageur et géographe Jean Morisset; ("Perspectives théoriques") ; ce chapitre introductif composé de trois essais est suivi de cinq chapitres centrés chacun sur un écosystème particulier : la toundra, la plaine, la forêt, le fleuve et le désert. Chaque chapitre se subdivise en un ou plusieurs essais écrits par des universitaires venus d'horizons divers (études littéraires québécoises, brésiliennes, littérature francophone, anglophone, littérature arabe et sémiotique). Centré sur des littératures de plusieurs aires géographiques, cet ouvrage propose des questions fondamentales qui lient la littérature de voyage, la poésie et la littérature environnementale. Il montre le caractère à la fois philosophique, sociétal et militant de ces textes de l'espace.

2 Après les trois essais qui constituent les "perspectives théoriques", un seul essai compose les chapitres consacrés à la toundra, à la plaine et au fleuve. Tandis que deux essais constituent les chapitres consacrés à la forêt et au désert. Le but de l'ouvrage est défini par ses directrices dès la première phrase de l'introduction : celui-ci "explore la géopoétique des confins au sens géographique du terme, en mettant au premier plan des paysages grandioses, ceux du désert, de la forêt, de la toundra, de la banquise, du fleuve, de la lande, autant d'espaces soumis aux forces vives des éléments et qui mettent le corps et l'esprit à l'épreuve. Là où la végétation prolifère de manière fulgurante, là où le rythme de l'eau anime le paysage, là où le minéral impose ses lois, là où le vent souffle à perdre haleine, là où les phénomènes premiers retiennent toute l'attention, les confins apparaissent", expliquent-elles, et dès cette introduction, apparaît le magnifique style de ce livre, poétique et profond (7). La géopoétique y est bien définie comme inscrivant "au cœur de ses préoccupations, le rapport à la terre au monde, aux choses" (7). L'ouvrage conduit le lecteur dans un voyage au cœur du Sahara, de l'Océan indien, de l'Ecosse, du Canada, de l'Amazonie et du Grand Nord entre autres. Les auteurs étudiés montrent un riche tour d'horizon avec deux écrivains québécois, Francine Ouellette et François Turcot ; un auteur libyen, Ibrahim Al Koni ; les écrivains voyageurs Kenneth White, Jean Morisset et Sylvain Tesson; deux femmes écrivaines voyageuses au tournant du XXe siècle, Isabelle Eberhardt et Marie Le Franc ; un écrivain voyageur surréaliste, Benjamin Péret ; deux écrivains du voyage "passionnés de déserts et d'océans", Pierre Loti et J.D. Le Clézio ; et l'écrivain sociologue brésilien Joao de Jesus Paes Loureiro ; les écrivains inuit Markoosie Patsauq, Zebedee Nungak et Mitiarjuk Nappaaluk et l'artiste graphique inuk Alootook Ipellie.

Dans le premier chapitre sur les "Perspectives théoriques", Rachel Bouvet définit "les paysages des confins : déserts, mers, forêts", et lance la réflexion sur les confins, affirmant que ce qui fait de ces paysages des confins, "c'est leur caractère inhospitalier" (19). Partant de la simple constatation géographique et quantitative que les déserts, mers et forêts "occupent $88 \%$ de la surface de la planète", elle glisse à la perception littéraire de ces éléments purement spatiaux qui génèrent depuis toujours "une rêverie de l'immensité et du lointain" (19). C'est "l'évolution des paysages des confins dans l'imaginaire occidental et leur construction au fil du texte" qu'étudie Rachel Bouvet (19). C'est ensuite à travers la pensée de Kenneth White, qui dénonce la coupure cartésienne de l'homme avec la terre, et notamment par l'analyse de son poème, "On Rannoch Moor", que Christophe Roncato-Tounsi montre le caractère militant de 
l'auteur et de ce poème et sa dimension écologique et le rattache aux écrivains environnementaux que sont Thoreau, Emerson, John Muir ou plus près de nous, Gary Snyder. Cette littérature de voyage-là, telle que la conçoit Kenneth White, n'a pas pour but l'évasion mais une "[reprise] de contact avec 'la terre originaire'" (52). Et c'est en cela que la démarche de White s'inscrit dans la philosophie environnementaliste. Avec l'essai de Rita Olivieri-Godet sur "l"espace évanoui' du continent américain", les textes étudiés, singulièrement, les Chants polaires de Jean Morisset (2003), étudient la construction d'un imaginaire "qui se réapproprie les références culturelles des Peuples Premiers et leurs formes de rapport au monde pour bousculer l'imaginaire occidental sur les confins et inaugurer de nouveaux questionnements identitaires" (63). L'auteur souligne "le 'rapport sensible et intelligent à la terre', 'l'appel du dehors' qui conduit à l'exploration physique et à une herméneutique des lieux, l'interrogation sur le rapport au monde sont autant d'éléments caractéristiques de la démarché géopoétique, qui est au centre de la réflexion-poétique de Jean Morisset" (64). En partant de la carte du XVIe siècle de la "Terra Hochelaga", représentation d'un lieu iroquoien constituée originairement d'un texte, de cartes et d'écorces d'arbres, l'étude suggère que "la parole poétique fait corps avec les éléments de la nature" (68) et montre "la grande mémoire orale, les archives de la nature", comme le dit Morisset (68). Cet essai, qui insiste sur "l'acte de remémoration" qui caractérise l'œuvre de Jean Morisset, suggère en quoi cette œuvre montre un auteur "attentif [...] à la mémoire orale des peuples, aux signes 'incrustés sur la terre et inscrits dans le visage des êtres' ${ }^{\prime} 1$ " (69). Le poète nomade fait de son nomadisme une plongée dans la conscience de la relation à la terre et de la connexion entre les peuples. Ses Chants polaires révèlent, à travers le mélange des langues - français, anglais, inuktitut - "des passages entre les mondes inuit et occidental" (75). A travers de belles analyses des poèmes de Morisset, l'auteur montre comment ces poèmes révèlent "la symphonie de l'univers et nous font faire l'expérience d'une autre façon de l'habiter" (82).

Ces paysages des confins qui passent par la lande écossaise vue par Kenneth White et le Grand Nord canadien parcouru par Jean Morisset, vont s'ouvrir sur "Les mots de la toundra : poétique du territoire dans la littérature inuit", analysés par Nelly Duvicq, qui étudie la toundra dans la géographie des Inuit et dans des œuvres littéraires, littérature orale ou romans, où les Inuit écrivent sur leur territoire. L'essai rappelle que ces textes évoquent l'assujettissement de l'homme aux lois de la nature. Se fondant sur des anciens récits et chants, sur le roman groenlandais d'Augo Lynge, écrit en 1930, Trois cents ans après, l'auteur de l'essai évoque le savoir topographique et "la pérennité de l'oralité inuit" (93). L'évocation de la marche dans la toundra donne lieu à l'observation de la végétation et aux sensations qui y sont liées, ce qui n'est pas sans rappeler le rôle de la marche chez Thoreau ou chez l'écrivaine amérindienne Linda Hogan. C'est "le corps de la toundra" qui, dans la poésie de Taqralik Partridge, montre "un univers rempli d'histoires à recevoir, à vivre et à partager'" (94). Dans ce paysage arctique défini par les géographes comme un "désert", "les Inuit voient l'abondance". Cette distinction entre le vide et le plein établie ici par Nelly Duvicq rappelle l'essai du romancier canadien Rudy Wiebe qui dans Playing Dead, parle de "pays nu" pour évoquer le paysage arctique dans la perception des explorateurs de l'expédition Franklin qui ne voient pas toute la vie contenue dans cet espace que leur montrent les Amérindiens. De la même manière, dit Nelly Duvicq, les Inuit montrent l'espace comme un "lieu saturé d'éléments, et d'êtres visibles et invisibles" (96). Est évoqué aussi dans cet essai le texte de John Weetaltuk, Land, écrit "en réaction à la signature de la 
Convention de la Baie James et du Nord québécois", texte qui est "devenu célèbre en tant que discours écologique précurseur" (100) ; le territoire n'est plus simplement un espace "avec lequel les Inuit partagent leur quotidien et avec lequel tantôt ils luttent tantôt ils coopèrent ; c'est aussi celui qu'ils peuvent perdre et dont ils saisissent alors la beauté et l'exception" (100). Poèmes comme textes politiques mettent en valeur une "réalité sensorielle collective" (103) et "l'énergie et la force de vie" (104) contenus dans ce paysage arctique.

5 Le chapitre suivant est consacré à la plaine et composé d'un essai de Licia Soares de Souza, "La géopoétique des confins de Francine Ouellette", romancière québécoise dont les deux romans analysés, Au nom du père et du fils (1984) et Le sorcier (1985), évoquent les conflits entre la culture canadienne blanche et les peuples des Premières Nations. L'analyse est enrichie de comparaisons avec la culture brésilienne dont l'auteur souligne "qu'au Brésil les entrecroisements entre Blancs, Amérindiens et descendants d'Africains sont si prégnants qu'ils sont plus que des représentations littéraires ; ils existent vraiment comme faits anthropologiques [...]" (111). En étudiant ce qu'elle nomme les "espaces-gigognes", les "modes d'emboîtements sémiotiques" et les "enchevêtrements de voix narratives" (122-123), Licia Soares de Souza voit "les fondations des identités-résistance" dans la représentation des espaces et dans ces fictions montrant à la fois la vie des Algonquins dans les Laurentides, la colonisation, les rapprochements et les conflits culturels ; elle lit une part importante de l'histoire du Canada, la lutte de Louis Riel pour les minorités, "dans la perspective d'un société multiculturelle basée sur le respect de l'Autre" (123). L'étude de la géopoétique des confins à travers les prairies vues dans des romans québécois souligne ainsi le rapport entre l'espace et l'histoire des peuples.

Le chapitre consacré à la forêt se compose de deux essais : le premier, d'Élise Lepage, "Émergence d'une subjectivité des confins : les premiers recueils de François Turcot", après avoir évoqué la notion de paysage, étudie les deux premiers recueils de l'auteur québécois, Miniatures en pays perdu (2006) que l'auteur définit comme "un apprentissage du regard" (141), et Derrière les forêts (2008), dans lequel est révélé le caché, l'invisible. La marche revient encore comme mouvement qui révèle la nature et les marcheurs sont rapprochés de personnages de contes pour enfants dans la mesure où ce recueil, dit l'auteur, réactive l'imaginaire de la forêt à la fois comme "chaosmos" (terme "forgé par Joyce dans Finnegans Wake et par lequel il désigne l'intrication de l'ordre et du désordre", précise l'auteur en note) et comme labyrinthe ; l'auteur se réfère aux Géographies imaginaires de Pierre Jourde pour évoquer "le lieu d'où nous venons", à la fois "origine géographique" et "mémoire ancestrale" (142-143).

Dans le second essai, de Leonor Lourenço de Abreu, "D'une forêt à l'autre: déambulations poétiques de Benjamin Péret", la forêt du poète surréaliste, à la fois "espace physique et paysage mental", apparaît comme "un creuset de transfiguration mythique" (162). Partant d'un texte publié dans la revue Minotaure en 1937, "la nature dévore le progrès et le dépasse", texte qui fait référence au fondateur du Service de Protection des Indiens (Candido Rondon), l'auteur évoque deux récits de voyage de Benjamin Péret, écrits après un voyage qu'il avait fait chez plusieurs peuples indigènes du centre du Brésil en 1956, Visite aux Indiens et Dans la zone torride du Brésil ${ }^{2}$. Une belle analyse du texte de Péret montre "la terre vue du ciel" où s'articulent une image du pays réel et "un pays(age) imaginaire" (152). On voit une nature dangereuse avec "la lutte des espèces pour la survie" (152). Dans une belle image prolongeant celle de 
Lévi-Strauss invitant à "jouer à saute-mouton par-dessus les étapes anciennes", l'auteur voit l'avion comme "un véritable deus ex machina des temps modernes [qui] permet de condenser les catégories spatiales et temporelles" (154). L'auteur évoque avec la forêt amazonienne la rencontre de deux mondes, la métaphorisation de l'inconscient, la rhétorique de l'altérité. S'appuyant sur "La lumière ou la vie", l'auteur montre que le poète présente la forêt comme un personnage "dévorateur" (156) dans un "climat d'inquiétante étrangeté" (157). La forêt tropicale du Brésil de Benjamin Péret présente "une dialectique de la vie et de la mort" (161). L'auteur de l'essai montre, à l'aide des textes du poète surréaliste français, comment "la forêt demeure l'espace privilégié où la pensée se trouve aux prises avec le magique et le mystérieux qui s'expriment symboliquement dans les constructions mythopoétiques des peuples indigènes".

8 Le chapitre sur le fleuve se compose d'un essai de Brigitte Thiérion, "Expérience poétique du grand fleuve chez João de Jesus Paes Loureiro. Proposition pour une lecture géopoétique". A travers l'analyse de l'ouvrage Au-delà du méandre de ce fleuve (2002), à la fois poème en prose, récit de voyage et fable, du poète, dramaturge et sociologue brésilien João de Jesus Paes Loureiro, l'auteur de cet essai montre le fleuve comme une entité naturelle incarnant la "tension entre mobilité et permanence", et une allégorie peignant une "démarche d'immigration et d'exil" (168). Le voyageur, le rameur et le fleuve sont les trois acteurs du drame. L'interpénétration entre le fleuve et l'homme est analysée à la lumière notamment de la vision bachelardienne de l'eau. Le poème montre la symbiose "qui résulte du contact étroit et prolongé entre l'homme et la nature" (170. Les frontières entre l'humain et le non-humain se diluent. Le langage du fleuve est analysé à la lumière des mythes, des légendes qui sont "portées par la langue des eaux, la langue du fleuve, à mesure que le voyageur progresse dans son voyage" (173). Le fleuve peint ici montre aussi la réalité dramatique de pans de terre érodée emportés par les crues et dans la supplique d'un personnage "mi-homme, mi-dieu", se lit "le drame écologique et humain qui frappe l'Amazonie" (178). L'essai montre aussi comment ce voyage débouche sur une perception esthétique de la nature dans le regard $\mathrm{du}$ voyageur, qui "se partage entre l'horreur et le merveilleux" (184). L'essai montre comment dans cette œuvre, l'univers du fleuve apparaît comme "un espace qui ne trouve sa réalité que dans l'imaginaire" (185) et en même temps, la démarche esthétique s'ouvre sur une réflexion écologique.

Le dernier chapitre concerne le désert et se compose d'un essai d'élisabeth Vauthier, "Entre roc et sable, le récit du Sahara comme lecture immémoriale de l'humain dans Le Saignement de la pierre d'Ibrahim Al Koni". Quittant l'Arctique et l'Amazonie, les littératures francophone, anglophone, inuit et brésilienne, ce dernier essai nous conduit au cœur du désert à travers une œuvre de littérature arabophone Le Saignement de pierre (Nazif-al-Hajar), du poète libyen Ibrahim Al Kûnî (Al Koni). Ce roman est analysé en tant que "roman du désert" et il apparaît comme "une réflexion sur l'homme et le sacré" où le désert joue un rôle prépondérant et où sont convoqués les "mythes méditerranéens et la culture africaine" (194). Dans la confrontation entre le personnage principal, nomade touareg qui a toujours vécu dans le désert, "dans le sillage d'un père fasciné par le mouflon, animal sacré, officiellement disparu de la région depuis le XVIIe siècle", et un "chasseur assoiffé de sang" (194) venu dans la région "devenue un sanctuaire pour les animaux" dans le seul but de tuer les mouflons qu'il pourrait trouver, et qui va se terminer par la mort du premier qui refuse de dévoiler le lieu où se cachent les derniers représentants de l'espèce, se lit la lutte entre 
le Bien et le Mal. Mais on y voit aussi un reflet d'une réalité tragique où les défenseurs d'espèces animales menacées paient leur engagement de leur vie (Dian Fossey ou Bruno Manser parmi les plus célèbres et beaucoup d'anonymes aussi). Comme c'était le cas dans les textes peignant le Grand Nord arctique, le désert n'apparait pas comme un lieu vide mais comme un espace qui "se constitue en élément vivant et signifiant", peuplé de multiples créatures, humaines, non humaines et fantastiques (195). Elizabeth Vauthier montre comment la "relation familiarité/étrangeté" est inversée. C'est le désert qui est familier et "le monde habité par les hommes", le monde des sédentaires "est renvoyé au domaine des confins, territoire de l'Autre, dysphorique, voire traumatisant" (196). Le Sahara génère "une réflexion sur la destinée humaine" qui s'inscrit dans un imaginaire ancien du désert. Il parle à la fois de réalité et d'espace mythique. A la fois ancré dans la réalité du Sahara libyen et dans "un lieu mythique qui retrace les origines", il montre l'un de ses habitants, le mouflon, à la fois comme une espèce menacée et comme un animal sacré d'une force redoutable, "esprit de la montagne" auquel le personnage principal "finit par faire allégeance, sacrifiant sa vie pour le protéger" (199). Le désert y est aussi à la fois un "conservatoire de la mémoire de ce monde" et un "refuge des êtres blessés par la violence des hommes" (201). Le désert saharien, tout en étant le lieu réel d'une vie intense et difficile, est aussi un lieu mythique et "une clef pour la compréhension de l'univers" et pour la relation à l'Autre, humain et non humain.

De la lande écossaise au Nunavut et de l'Amazonie au Sahara, ce bel ouvrage collectif conduit le lecteur dans un voyage au cœur de récits de voyages, de poèmes, d'essais, de chants aussi, qui donnent à lire le rapport de l'homme au monde, à la nature, à l'Autre, humain et non humain. Voyage poétique et anthropologique à dimension écologique, cette "Géopoétique des confins" fait de ces espaces réputés inhospitaliers les lieux de notre relation au monde et aussi de nos paysages intérieurs. Voyage poétique, philosophique et politique, l'ouvrage magistralement dirigé par Rachel Bouvet et Rita Olivieri-Godet, nous conduit, dans le fil lumineux qui tisse tous les espaces et toutes les œuvres évoqués, dans les écosystèmes du monde par le prisme des poètes, des romanciers, des voyageurs. C'est un ouvrage fondamental et beau qui passionnera tous ceux et toutes celles qui s'intéressent à la littérature de voyage mais aussi à la poésie, aux textes environnementaux, à l'écologie et au rapport entre texte et paysage. C'est un cheminement magnifique au cœur du monde et des littératures du monde.

\section{NOTES}

1. Jean Morisset, Récits de la terre première, Montréal: Lemeac, 2000, 107.

2. "Visites aux Indiens" a été réédité en 2014 sous le titre Dans la zone torride du Brésil, Nolay: Les éditions du chemin de fer, 2014.

3. Claude Lévi-Strauss, Tristes tropiques [1955], Paris: Plon, coll. "Terre humaine", 1995, 126. 
INDEX

Keywords : geopoetics, travel writing, poetry, ecology, environmental literature, human/ nonhuman relationship, landscape, desert, river, forest, tundra, plain; Indigenous peoples, First Nations, Amazonia, Arctic, Sahara, Scotland, Brazil, Canada, Nunavut, language

Mots-clés : géopoétique, littérature de voyage, poésie, littérature environnementale, écologie, relation humain/non humain, paysage, désert, fleuve, forêt, toundra, plaine, peuples autochtones, Premières Nations, Amazonie, Arctique, Sahara, Ecosse, Brésil, Canada, Nunavut, langage

\section{AUTEURS}

\section{FRANÇOISE BESSON}

Professeur émérite

Université de Toulouse - Jean Jaurès

francoise.besson@univ-tlse2.fr 